\title{
ORIGINAL RESEARCH \\ A Large and Giant Bifurcation Aneurysm Model in Canines: Proof of Feasibility
}

R. Ysuda

C.M. Strother

B. Aagaard-Kienitz

K. Pulfer

D. Consigny
BACKGROUND AND PURPOSE: To our knowledge, no reproducible animal model of a giant bifurcation type aneurysm has been described. It was our aim to develop a 1-stage and reproducible model of a venous pouch giant aneurysm in canines.

\begin{abstract}
MATERIALS AND METHODS: Nine canines were involved. Bilateral CCAs were exposed. The left CCA was divided and its distal segment was swung to the right side. Using the right CCA and the distal segment of the left CCA, either a bifurcation or a terminal arterial structure was constructed. Bilateral external jugular veins were also exposed. A 30-mm vein segment was harvested from each side. Each vein graft was split and unfolded to make 2 venous sheets. These sheets were then joined top-tobottom so as to form a single cylinder in such a way that original adventitial side of the venous sheets was on the exterior surface. In 2 instances, pieces of polytetrafluoroethylene were employed along with the venous sheets. The combined vein graft was then incorporated into the arterial anastomosis. Lastly, the top of the venous pouch was closed. No medications for anticoagulant or antiplatelet were used throughout the study period. Follow-up imaging studies were performed.
\end{abstract}

RESULTS: It took 2.5 hours on average for 2 operators to create an aneurysm. Eight of the 9 aneurysms were patent at follow-up. The cause of the spontaneous thrombosis was unclear despite autopsy. All the aneurysms had a maximum diameter $>20 \mathrm{~mm}$.

CONCLUSIONS: We demonstrated and illustrated a 1-stage and reproducible procedure to create a model of a venous pouch bifurcation giant aneurysm in canines.

ABBREVIATIONS: CCA $=$ common carotid artery; $E J V=$ external jugular vein; $\mid \mathrm{A}=$ intra-arterial; PTFE = polytetrafluoroethylene; VOR = volume to the area of the ostium
E xperimental aneurysm models in animals have played an important role in the development and preclinical evaluation of endovascular devices and techniques for treatment of intracranial aneurysms. While many experimental aneurysm models have been reported by using canines, rabbits, swine, and rodents, ${ }^{1-4}$ a recent systematic review of in vivo experimental aneurysm models demonstrated that the most useful models for preclinical testing on aneurysm-treatment devices were the elastase-induced aneurysms in rabbits and the venous pouch aneurysms in dogs. ${ }^{5}$ Since its introduction by Cloft et $\mathrm{al}^{6}$ and Altes et $\mathrm{al},{ }^{7}$ the rabbit elastase-induced aneurysm, by using the stump of the right CCA, has been widely used. ${ }^{8,9}$ Several studies focusing on natural history of the aneurysms proved the feasibility of the aneurysm model with long-term patency. ${ }^{10,11}$ On the other hand, since the publication by German and Black, ${ }^{1}$ canine venous pouch aneurysms have also been commonly utilized. ${ }^{12,13}$ Their natural history was also studied and they also showed long-term patency, with few spontaneous thromboses or ruptures. ${ }^{14-16}$

Giant aneurysms are still challenging for both traditional surgical and endovascular treatment. ${ }^{17-19}$ Therefore, further efforts to improve endovascular devices and techniques for

Received March 11, 2011; accepted after revision June 16.

From the Department of Radiology (R.Y., C.M.S., B.A.-K., K.P., D.C.), University of Wisconsin School of Medicine and Public Health, Madison, Wisconsin; and Graduate School of Medicine (R.Y.), Mie University, Tsu, Mie, Japan.

Previously presented in part at: Annual Meeting of the Society of Neurolnterventional Surgery (SNIS), Carlsbad, California, July 26-29, 2010

Please address correspondence to Charles M. Strother, Department of Radiology, University of Wisconsin School of Medicine and Public Health, E3/366 Clinical Science Center, 600 Highland Ave, Mail Code 3252, Madison, WI 53792-3252; e-mail: Strother@wisc.edu http://dx.doi.org/10.3174/ajnr.A2789 such aneurysms, and thus, in turn, development of suitable animal models, is warranted. While both elastase-induced and venous pouch aneurysms have potential to grow, ${ }^{10,15}$ neither result in aneurysms that are large enough to adequately simulate giant aneurysms (the final [maximum] size was reported as $10.0 \pm 2.2 \mathrm{~mm}$ for the rabbit elastase-induced aneurysms $\mathrm{s}^{10}$ and $19.3 \pm 1.3 \mathrm{~mm}$ for the canine venous pouch aneurysms). ${ }^{15}$

In an attempt to develop an animal model of a larger aneurysm, Ding et al succeeded in controlling aneurysm size in the rabbit elastase-induced aneurysm by adjusting the position of ligation $^{20}$ and, more recently, by creation of a presurgical arteriovenous fistula. ${ }^{21}$ The maximum size of an aneurysm created in these reports, however, was $15.3 \mathrm{~mm}$ and $16.8 \mathrm{~mm}$, respectively, still too small for a giant criterion. Several attempts have also been made in canines to create a giant venous pouch aneurysm model. To the best of our knowledge, only 4 reports where a venous graft of $20 \mathrm{~mm}$ or longer was used for aneurysm creation are available. ${ }^{22-25}$ In 2 of these, aneurysm creation required cumbersome 2 -staged procedures, ${ }^{22,23}$ and in 1 , it required intraoperative heparin injection and daily aspirin intake after surgery. ${ }^{25}$ In addition, all the aneurysms created in these studies were of the sidewall type. While giant aneurysms mainly occur at the internal carotid artery, mostly in a sidewall geometry, they are also not infrequently found at the middle cerebral artery, the anterior communicating artery, and the basilar apex, as either a bifurcation or a terminal geometry. ${ }^{26}$

On the basis of these observations, our purpose was to develop a 1-stage and reproducible procedure that does not require any use of anticoagulant or antiplatelet agents, which would create a venous pouch giant aneurysm model in canines. Such a model should be useful for preclinical evaluation 


\begin{tabular}{|c|c|c|c|c|c|}
\hline Dog \# & Artery & "Aneurysm" Sac & $1 s t \mathrm{f} / \mathrm{u}$ & 2nd $f / u$ & Comment \\
\hline 1 & Bifurcation & Vein graft & IV (2) & $\mathrm{IA}(4)$ & \\
\hline 2 & Bifurcation & Vein graft & IV (1) & IA (3) & \\
\hline 3 & Bifurcation & Vein graft & IV (1) & IA (3) & \\
\hline 4 & Bifurcation & Vein graft & IV (3) & IA (6) & \\
\hline 5 & Bifurcation & Vein graft & IV (3) & $\mid A(6)$ & \\
\hline 6 & Bifurcation & Vein graft & IV (3) & IA (12) & An aneurysm was thrombosed \\
\hline 7 & Bifurcation & Vein graft & IV (3) & $\mid A(6)$ & \\
\hline 8 & Bifurcation & Vein graft + PTFE & IA (2) & $*$ & Dog was sacrificed after the $1 \mathrm{st} f / \mathrm{u}$ due to sickness \\
\hline 9 & Terminal & Vein graft + PTFE & IV (3) & IA (7) & \\
\hline
\end{tabular}

Note:-IV and IA represent a modality employed for the follow-up study. A number in parentheses represents the interval in weeks between aneurysm creation and each follow-up study. Animals were sacrificed on the day following the 2nd follow-up. f/u indicates follow-up study; IV, intravenous digital subtraction angiogram; IA, intra-arterial digital subtraction angiogram.

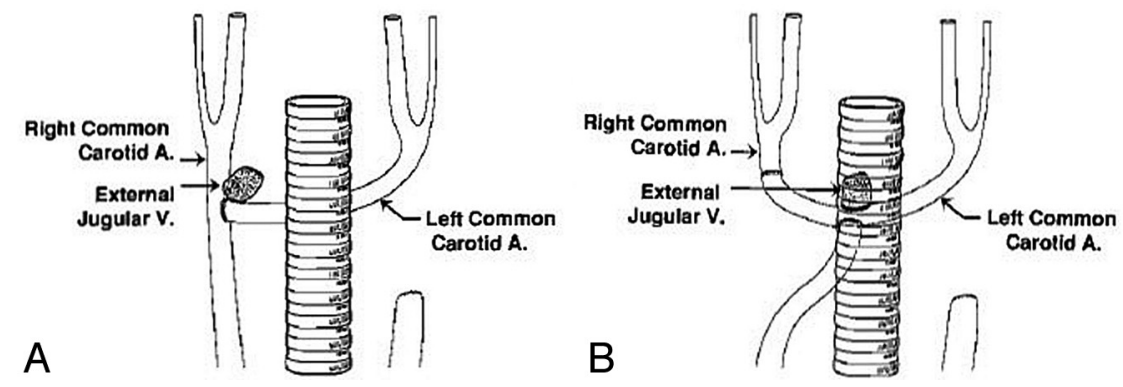

Fig 1. Arterial structures of $(A)$ bifurcation and $(B)$ terminal. Adapted from Strother, Graves, and Rappe. ${ }^{28}$

of endovascular techniques and devices, as well as for hemodynamic studies to address giant aneurysms. This report describes and illustrates this model.

\section{Materials and Methods}

Nine mongrel dogs were involved in this study. All the procedures were performed under a protocol approved by the University of Wisconsin Animal Care Committee. Canines were 9-11 kg in weight and were maintained with a standard laboratory diet. After an overnight fast, general anesthesia was induced with $10 \mathrm{mg} / \mathrm{kg}$ of propofol IV injection and was maintained with $1 \%-5 \%$ isoflurane and $100 \%$ oxygen via endotracheal tube. The neck region was prepared and draped in a sterile manner. No anticoagulant or antiplatelet was used before and during the surgery.

\section{Artery Construction}

Either a bifurcation or a terminal arterial structure was constructed (Table 1) using an established surgical procedure previously reported from our laboratory. ${ }^{27,28} \mathrm{In}$ brief, a paramedian 8 -cm long skin incision was made bilaterally, and CCAs were exposed after gentle muscle separation. The left CCA was ligated proximally while a temporary vascular clamp was applied distally. The left CCA was cut just above the proximal ligation. A tunnel was made beneath the trachea and the distal segment of the left CCA was swung to the right side passing through the tunnel. For creation of the bifurcation geometry, an arteriotomy was made on the midportion of the right CCA after the proximal and the distal clamps were applied, and the distal left CCA was sewn end-to-side into the right CCA (Fig 1A). For creation of the terminal model, the right CCA was divided and its distal portion was anastomosed end-to-end with the distal left CCA. The proximal portion of the right CCA was then anastomosed end-to-side into the undersurface of the $\mathrm{U}$ formed by this linkage (Fig $1 B$ ).

\section{“Aneurysm” Sac Creation}

Bilateral EJVs were isolated and ligated proximally and distally. A 30-mm length segment was harvested from each side (Fig 2A) and then rinsed with heparinized saline (1000 $\mathrm{U}$ heparin/100 mL saline). Care was taken to harvest the veins from portions of the jugular vein having no valves. Each of the 2 segments was sliced longitudinally and unfolded to make 2 venous sheets (Fig $2 B$ ). For creation of a bifurcation aneurysm, 1 end of these sheets was trimmed into a shape that would fit into the arterotomy made in the anastomosed right and left CCAs (Fig 2C). The 2 venous segments were then joined top-tobottom so as to form a single cylinder. To facilitate this, a handmade fixture was employed, which consisted of a single rubber cylinder mounted on a metal axle, which was connected to 2 support stands (Fig $2 D$ ). The 2 vein sheets were mounted onto the rubber bar in such a way that the original adventitial side of each of the 2 sheets was on the exterior surface (Fig 2D). In 2 instances, to create a wider aneurysm, small pieces of PTFE were interleaved between the 2 vein sheets (without expanding the surgical field, it was impossible to harvest additional segments of autologous vein long enough to supplement the initial vein sheets). After the venous sheets were sewn together, 1 of the metal axle ends was detached from the stand, and then the combined vein graft was carefully positioned toward the arteriotomy site. In incorporating the vein graft into the arterial anastomosis, the graft remained on the rubber bar so as to keep the graft cylindrical and thus simplify suturing the vein to the arteries (Fig $3 A$ ). After the vein was connected to the arteries, the support fixture was withdrawn and the top of the venous pouch was closed with sutures (Fig 3B). All of the sutures were performed in a running manner with 7-0 Prolene (Ethicon, Somerville, New Jersey). All of the clamps were then removed and, if any leak was observed, additional sutures were applied until hemostasis was obtained (Fig 3C). Before closing the surgical wounds, gentle digital dilation was made into retrotracheal space to create adequate space for the aneurysm sac (this does not need to be 


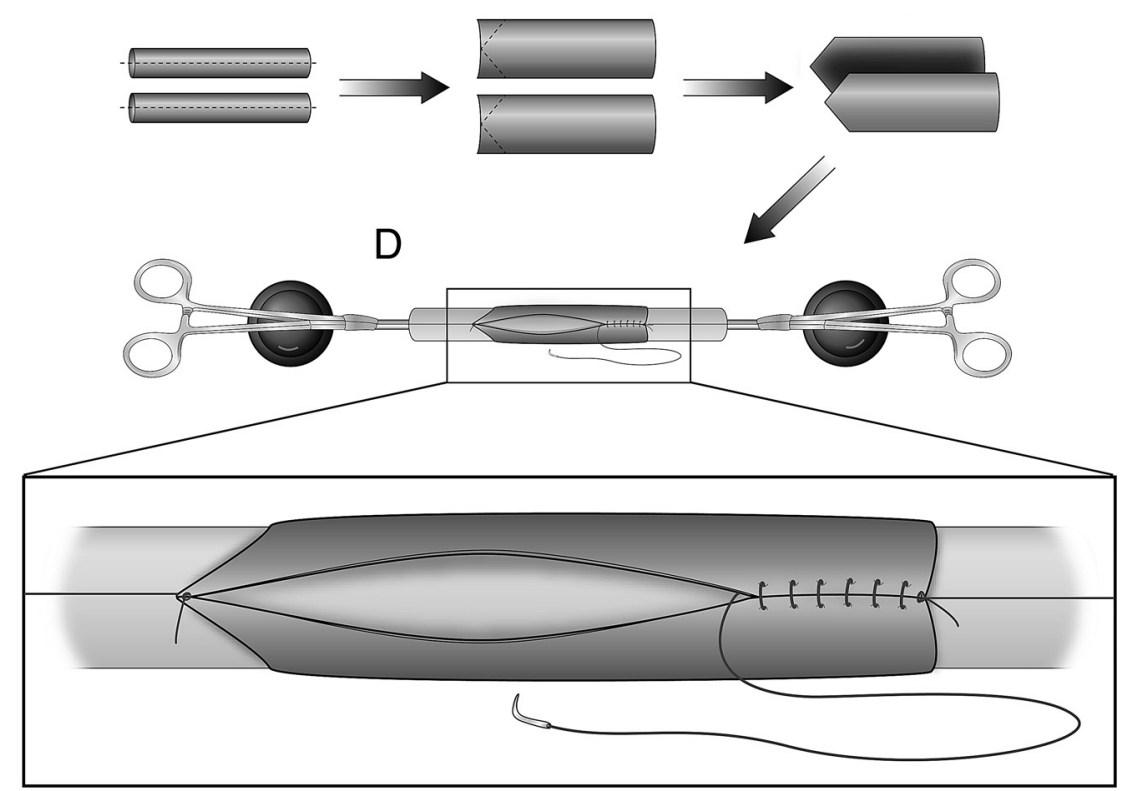

Fig 2. Bilateral external jugular veins are harvested. $A$, The vein grafts are split to make 2 venous sheets. $B$, These sheets are trimmed into a shape that would fit into the bifurcation arterial structure. The sheets are then mounted on a device, shown in $D$, in a way such that the original adventitial side is on the exterior surface. C, A handmade device to facilitate venous pouch preparation. Note that, by using 2 clamps, tension is made on the suture line to facilitate the procedure.
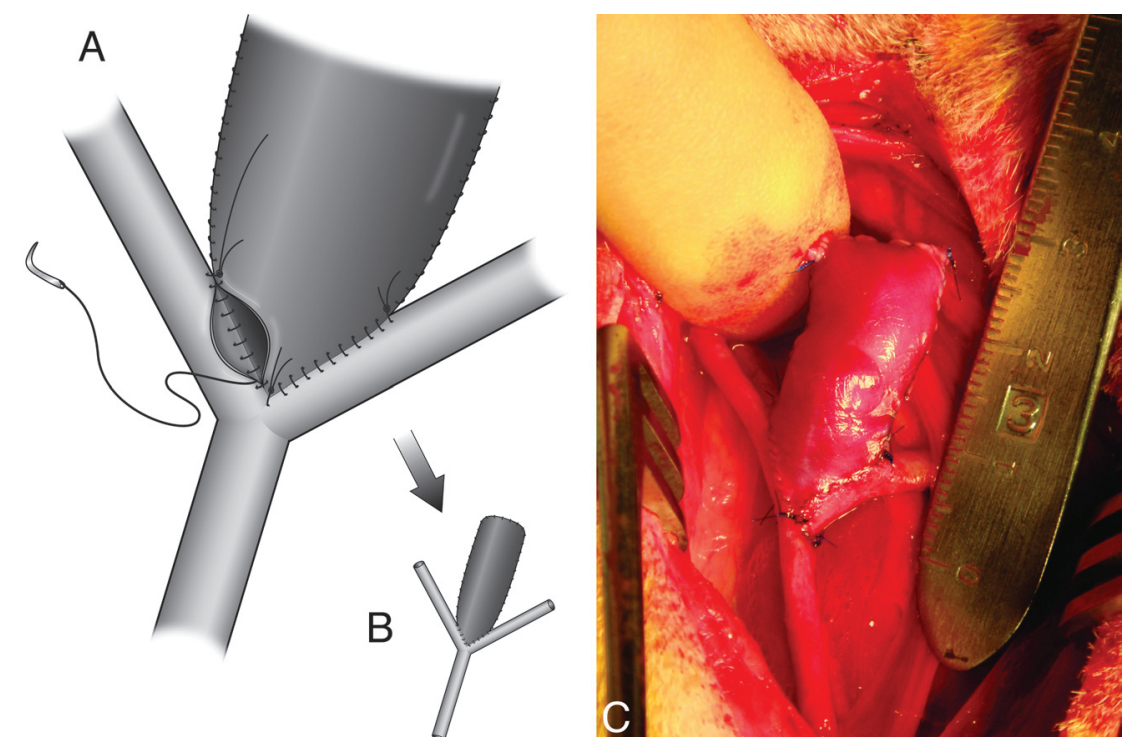

Fig 3. Incorporation of the venous pouch into the arterial structure. $A$, At this point, the venous pouch still remains on the device shown in Fig $2 D$, so as to keep the venous pouch cylindrical and thus facilitate suturing. $B$, The top of the venous pouch is also sutured. $C$, Completion of "aneurysm" creation after achieving hemostasis.

done as a part of the routine used to create standard venous pouch aneurysms). In the closure, special attention was paid not to compress the "aneurysm" with surrounding tissue. Fascia and skin closure were achieved by using 3-0 Prolene in multiple layers.

\section{Follow-Up Study}

The animals were maintained under observation until the follow-up study. No anticoagulant or antiplatelet was used after the surgery. Two follow-up studies were performed in 8 of the 9 animals. One animal developed internal bleeding 2 weeks after surgery. To assess the cause of this bleeding, this animal underwent emergency IA angiography; the aneurysm was patent (Fig $4 H$ ) and no cause for the bleeding was determined. At the advice of the attending veterinarian, this animal was euthanized and thus did not have a second follow-up study. The first follow-up study was performed in 8 animals by using an IV-DSA. The second angiographic follow-up was performed at intervals ranging between 2 and 9 weeks after the first follow-up by using standard IA-DSA techniques (Table 1). An IV study was done for the first follow-up because it was less invasive than an IA study. In our experience the IV studies provide images that are very comparable with an IA study.

Biplane DSA and 3D-DSA in all the subjects were acquired by using biplane C-arm angiographic systems (Artis zeego; Siemens Healthcare, Erlangen, Germany). Using a commercial workstation (Leonardo; Siemens Healthcare), the 3D-DSA data were reconstructed to volume-rendered 3D images. From the reconstructed 3D 

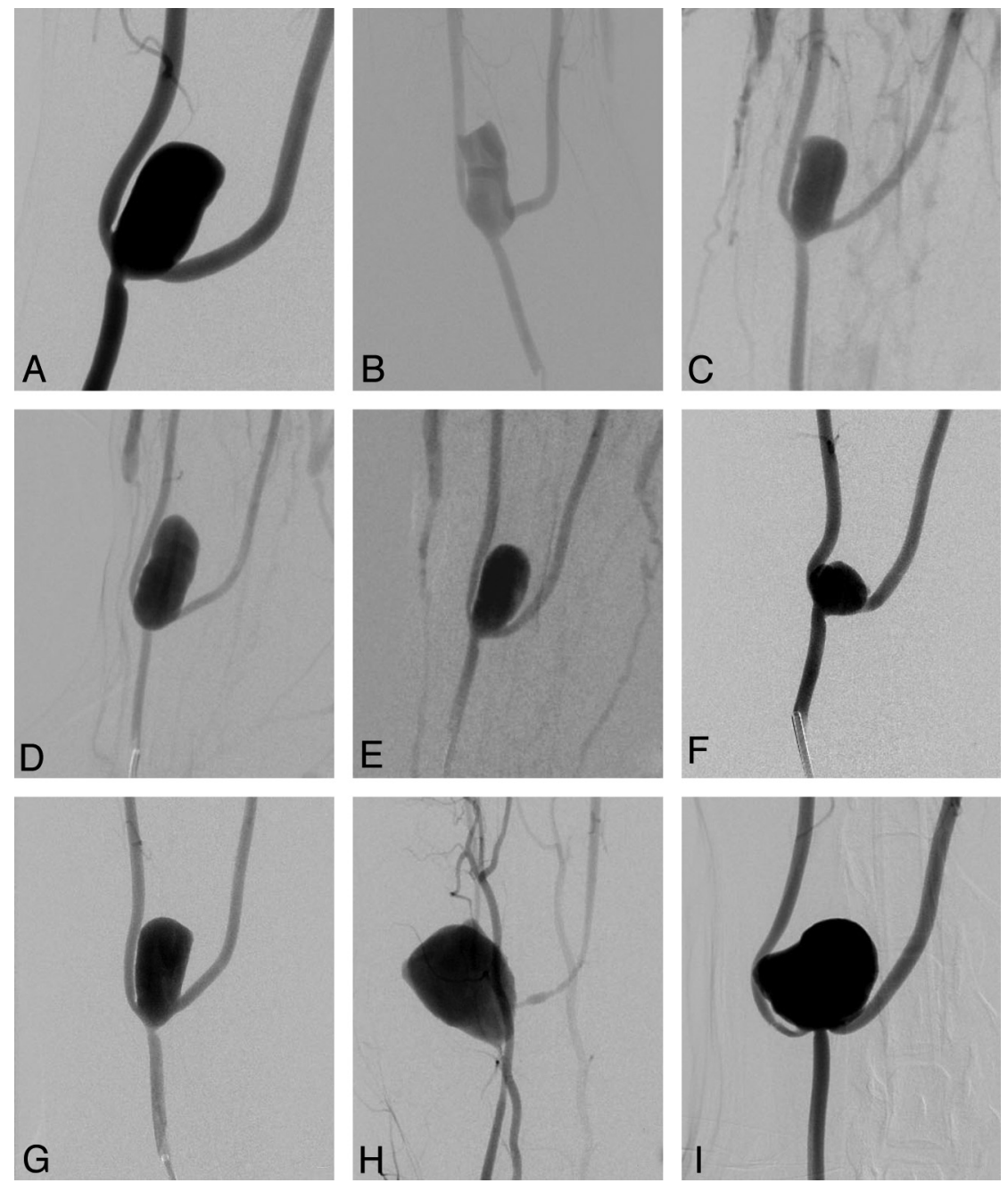

Fig 4. Images acquired at the second follow-up angiogram. $A, B$, and $C$ represent images of aneurysms from dogs 1,2 , and 3 , respectively. Except in 1 case $(F$, dog 6$)$, all the aneurysms remain patent. In cases $H$ and / (dogs 8 and 9), PTFE was used along with vein grafts.

images, aneurysm height, neck width, dome width, volume, and the area of the ostium were measured. The detail in measuring aneurysm volume and the area of the ostium was previously described elsewhere. ${ }^{29}$ Measurements of aneurysm volume and the ostium area were only performed on the second follow-up studies.

\section{Results}

It took 2.5 hours on average for 2 operators to create a giant aneurysm; while 1 operator (D.C.) constructed the arterial anastomosis, the other (R.Y.) prepared the venous pouch. No surgery-related complications occurred.

The first follow-up DSA revealed that in 8 of the 9 dogs, a giant aneurysm was successfully created; of the animals having a second follow-up angiogram, all 7 with patent aneurysms on the first follow-up were found to have patent aneurysms without any evidence of intra-aneurysmal thrombosis. (Fig $4 A-E$, $G-I$ ). The dimensions of the aneurysms were tabulated (Table 2 ). The mean value of all heights, dome widths, and neck widths was larger at the second follow-up. Because of the difference in imaging techniques used for the follow-up studies, the small sample size, and the variable time intervals between the follow-up studies, we cannot draw conclusions regarding the natural history of these aneurysms.
For the thrombosed aneurysm, the second follow-up imaging and autopsy were performed 12 weeks after the creation. No recanalization was observed on IA-DSA (Fig 4F). At the autopsy, the aneurysm was found widely open at the ostium, but most of its dome was filled with organized clot. However, no more clues to the cause of the thrombosis were extracted.

\section{Discussion}

We describe a reproducible technique by using vein grafts (supplemented by PTFE in 2 instances), to create a canine bifurcation giant experimental aneurysm model in a 1-stage procedure without any use of anticoagulation or antiplatelet medications.

Our series included aneurysms whose maximum diameter was between 20 and $25 \mathrm{~mm}$, whereas a giant aneurysm is commonly defined as greater than $25 \mathrm{~mm}$ in diameter. ${ }^{17-19,26}$ In some small canines the soft tissue space available to accommodate an aneurysm, combined with the size of the available arteries and veins, may make it impossible to create an aneurysm $25 \mathrm{~mm}$ or greater in size. Still, because the problems associated with the endovascular treatment of aneurysms with a maximum diameter between 20 and $25 \mathrm{~mm}$ are quite similar to those encountered in management of a true "giant" 


\begin{tabular}{|c|c|c|c|c|c|c|c|}
\hline \multirow[b]{2}{*}{ Dog \# } & \multicolumn{3}{|c|}{ Height (mm) } & \multirow[b]{2}{*}{ Dog \# } & \multicolumn{3}{|c|}{ Neck Width (mm) } \\
\hline & $1 \mathrm{st} \mathrm{f} / \mathrm{u}$ & 2nd f/u & Dif. & & $1 s t \mathrm{f} / \mathrm{u}$ & 2nd f/u & Dif. \\
\hline 1 & 19.0 & 20.0 & 1.0 & 1 & 8.9 & 9.9 & 0.9 \\
\hline 2 & 25.2 & 25.1 & -0.2 & 2 & 9.6 & 10.6 & 1.0 \\
\hline 3 & 21.7 & 24.1 & 2.4 & 3 & 8.6 & 9.6 & 1.1 \\
\hline 4 & 30.1 & 31.5 & 1.4 & 4 & 11.0 & 13.2 & 2.3 \\
\hline 5 & 22.3 & 21.7 & -0.6 & 5 & 8.4 & 8.3 & -0.1 \\
\hline 6 & $*$ & $*$ & $*$ & 6 & $*$ & $*$ & $*$ \\
\hline 7 & 22.9 & 23.2 & 0.3 & 7 & 8.8 & 9.0 & 0.2 \\
\hline 8 & 21.6 & * & $*$ & 8 & 10.4 & * & * \\
\hline 9 & 20.9 & 20.1 & -0.8 & 9 & 8.5 & 7.7 & -0.8 \\
\hline Mean & 23.0 & 23.7 & & Mean & 9.3 & 9.8 & \\
\hline \multirow[t]{2}{*}{ SD } & 3.4 & 4.0 & & SD & 1.0 & 1.8 & \\
\hline & \multicolumn{3}{|c|}{ Dome Width (mm) } & & \multicolumn{3}{|c|}{ Volume $\left(\mathrm{mm}^{3}\right) / 0$ stium Area $\left(\mathrm{mm}^{2}\right)$} \\
\hline Dog \# & $1 \mathrm{st} \mathrm{f} / \mathrm{u}$ & 2nd $f / u$ & Dif. & Cases & Volume & Ostium Area & VOR \\
\hline 1 & 10.1 & 11.4 & 1.4 & 1 & 1552.0 & 54.8 & 28.3 \\
\hline 2 & 10.5 & 11.8 & 1.3 & 2 & 2576.5 & 90.4 & 28.5 \\
\hline 3 & 10.5 & 12.4 & 1.9 & 3 & 2319.9 & 95.8 & 24.2 \\
\hline 4 & 13.4 & 14.4 & 1.0 & 4 & 4595.0 & 172.3 & 26.7 \\
\hline 5 & 10.9 & 12.2 & 1.3 & 5 & 2287.5 & 68.5 & 33.4 \\
\hline 6 & * & * & * & 6 & * & * & $*$ \\
\hline 7 & 12.1 & 12.2 & 0.1 & 7 & 2601.2 & 89.2 & 29.2 \\
\hline 8 & 18.6 & * & * & 8 & 2418.1 & 64.1 & 37.7 \\
\hline 9 & 20.7 & 22.8 & 2.1 & 9 & 3350.3 & 73.4 & 45.7 \\
\hline Mean & 13.3 & 13.9 & & Mean & 2712.6 & 88.6 & 31.7 \\
\hline SD & 4.1 & 4.0 & & SD & 906.3 & 36.7 & 7.0 \\
\hline
\end{tabular}

Note:-Volume and the area of the ostium were measured only for the second follow-up study.

* For dog 6, no measurements were performed because of spontaneous thrombosis. f/u indicates follow-up; Dif., difference.

aneurysm (25 mm or greater), ${ }^{18,19}$ we believe that the aneurysms created and described in this report would serve as suitable surrogates for a "giant" aneurysm.

In this study, the mean value of all the aneurysm heights, dome widths, and neck widths was larger at the second follow-up compared with those at the first (Table 2). Although it has already been reported that canine venous pouch bifurcation aneurysms grew over time, ${ }^{15}$ it is impossible, from our observations, to conclude that our venous pouch giant aneurysms may also enlarge. Because of the following reasons, we did not perform statistical comparison of the measurements between the follow-up studies; first, and most importantly, IV-DSA was employed for the first follow-up, while IA-DSA was employed for the second. Because, compared with IADSA, IV-DSA tended to result in less, and occasionally inadequate, contrast concentration in filling an aneurysm, the aneurysm dimensions could be underestimated on the IV-DSA study. Thus, for the second follow-up study, an IA-DSA with a 3D-DSA acquisition was used. In our opinion, this provides the greatest spatial and temporal resolution of any available imaging technique. For the IA-DSA studies, contrast was injected from a catheter placed in the vicinity of the aneurysm. Therefore, an aneurysm could be dilated by injection pressure, thus leading to an inaccurate measurement. Second, measurement of 2D parameters, such as aneurysm height, dome width, and neck width, highly depend on the projection from which they are observed. ${ }^{29}$ This may explain why some measurements at the second follow-up were smaller than those at the first. Third, the follow-up interval varied significantly.

Spontaneous thrombosis occurred in only 1 of the 9 animals. Kerber et al demonstrated that the use of preoperative aspirin made no difference in the patency rate of experimental aneurysms. ${ }^{24}$ Learning from the experience of Black and German, who established a relationship between experimental aneurysm thrombosis and the ratio of aneurysm VOR, ${ }^{30}$ in our study, we tried to make the ostium as large as possible when creating an aneurysm. More than 300 canine venous pouch bifurcation aneurysms have been created in our laboratory, and the diameters of the ostium usually have been adjusted to $6 \mathrm{~mm}$, compared with an aneurysm height of $10 \mathrm{~mm}$ (we have no data as to the aneurysm volume in most of these aneurysms). ${ }^{14}$ In contrast, in this study, the diameter of the ostium was adjusted to $10 \mathrm{~mm}$. Still, in 6 of the 8 aneurysms with 2 follow-up studies, the VOR was higher than the value of 28.3, above which, in Black and German's report, all experimental aneurysms thrombosed spontaneously. ${ }^{30}$ The difference between their results and ours is likely because of the difference of parent artery-aneurysm geometry (sidewall versus bifurcation or terminal), which significantly affects intra-aneurysmal hemodynamics. ${ }^{31}$ We believe further investigation for flow analysis (eg, computational fluid dynamics study) by using our model would be helpful for understanding a part of the natural history of giant aneurysms. Further study, however, is necessary to determine the value of using this model to further the understanding of the hemodynamics of very large and giant aneurysms in humans.

In 2 cases, PTFE was used to achieve a larger dome width. If $60 \mathrm{~mm}$ or longer vein segments were available, there was no need for the use of PTFE to create a wider aneurysm. However, in our animals, because of the position of the EJV (lateral in the operative field) and the desire to limit soft tissue damage ( kin incision was limited to $8 \mathrm{~cm}$ ), it was too hard to harvest 
such larger venous segments. Also, the vein graft tended to shrink after the harvest. Nonetheless, all the aneurysms in which PTFE was incorporated were patent at last follow-up (Fig $4 H$-, I). Additionally, no PTFE-related complication was observed during the study period. Because PTFE has been widely used as patch material for carotid endarterectomy with competitive long-term performance to vein patch, ${ }^{32}$ we believe it was a reasonable substitute for vein grafts. Once its long-term feasibility for in vivo experimental aneurysms is proved, it might be possible to replace vein grafts entirely with PTFE (and if a "ready-made" PTFE pouch becomes available, there is no longer need to adjust the size and suture in making an "aneurysm" sac). More experience with PTFE as a graft material for use in making aneurysms of this type is required.

Our model has several limitations. The "aneurysm" wall made up of venous tissue, with or without PTFE, does not reproduce histologic characteristics and biologic behavior of human cerebral aneurysm wall. Physiologic reaction to suture lines could also compromise histopathologic evaluation. These shortcomings specific to venous pouch aneurysms, however, should be accepted, as it is, so far, impossible to create a model of elastase-induced giant aneurysms. ${ }^{10,20,21}$ In relation to biologic behavior, a created aneurysm is not surrounded with CSF. Second, the model is not appropriate to study aneurysm initiation. Third, the cause of spontaneous thrombosis in 1 aneurysm was not clear. We usually do not perform any imaging studies on the day of aneurysm creation because patency of an aneurysm can be confirmed macroscopically (Fig 3C). If performed, however, further study (eg, flow analysis) might have provided some solutions. Fourth, the follow-up term was limited up to 7 weeks. Long-term followup, with special attention to PTFE, should be done.

Despite these limitations, we did show a method to reproducibly create a canine venous pouch bifurcation giant aneurysm model, and its feasibility. Our model would be useful for not only preclinical evaluation of endovascular devices and technique but also for flow analysis to address giant aneurysms.

Disclosures: Beverly Aagaard-Kienitz—Research Support (including provision of equipment or materials): Micrus Endovascular, Details: co-Pl of the Cerecyte Coil Registry; Consultant: Micrus Endovascular, MicroVention Terumo, Details: No financial remuneration accepted.

\section{References}

1. German WJ, Black SP. Experimental production of carotid aneurysms. N Engl J Med 1954;250:104-06

2. Cawley CM, Dawson RC, Shengelaia G, et al. Arterial saccular aneurysm model in the rabbit. AJNR Am J Neuroradiol 1996;17:1761-66

3. Hashimoto N, Handa H, Hazama F. Experimentally induced cerebral aneurysms in rats. Surg Neurol 1978;10:3-8

4. Massoud TF, Ji C, Guglielmi G et al. Experimental models of bifurcation and terminal aneurysms: construction techniques in swine. AJNR Am J Neuroradiol 1994;15:938-44

5. Bouzeghrane F, Naggara O, Kallmes DF, et al and The International Consortium of Neuroendovascular Centres. In vivo experimental intracranial aneurysm models: a systematic review. AJNR Am J Neuroradiol 2010;31:418-23
6. Cloft HJ, Altes TA, Marx WF, et al. Endovascular creation of an in vivo aneurysm model in rabbits. Radiology 1999;213:223-28

7. Altes TA, Cloft HJ, Short JG, et al. 1999 ARRS Executive Council Award Creation of saccular aneurysms in the rabbit: a model suitable for testing endovascular devices. American Roentgen Ray Society. AJR Am Roentgenol 2000;174:349-54

8. Dai D, Ding YH, Kadirvel R, et al. A longitudinal immunohistochemical study of healing of experimental aneurysms after embolization with platinum coils. AJNR Am J Neuroradiol 2006;27:736-41

9. Fujiwara NH, Kallmes DF. Healing response in elastase-induced aneurysms after embolization with a new platinum coil system. AJNR Am J Neuroradiol 2002;23:1137-44

10. Fujiwara NH, Cloft HJ, Marx WF, et al. Serial angiography in an elastaseinduced aneurysm model in rabbits: evidence for progressive aneurysm enlargement after creation. AJNR Am J Neuroradiol 2001;22:698-703

11. Ding YH, Dai D, Lewis DA, et al. Long term-patency of elastase-induced aneurysm model in rabbits. AJNR Am J Neuroradiol 2006;27:139-41

12. Graves VB, Partington CR, Rüfenacht DA, et al. Treatment of carotid artery aneurysms with platinum coils: an experimental study in dogs. AJNR Am J Neuroradiol 1990;11:249-52

13. Turk AS, Luty CM, Carr-Brendel V, et al. Angiographic and histological comparison of canine bifurcation aneurysms treated with first generation Matrix and standard GDC coils. Neuroradiol 2008;50:57-65

14. Turk AS, Aagaard-Kienitz B, Niemann D, et al. Natural history of the canine vein pouch aneurysm model. AJNR Am J Neuroradiol 2007;28:531-32

15. Tsumoto T, Song JK, Niimi $\mathrm{Y}$, et al. Interval change in size of venous pouch canine bifurcation aneurysms over 10-month period. AJNR Am J Neuroradiol 2008;29:1067-70

16. Kallmes DF, Altes DA, Vincent DA, et al. Experimental side-wall aneurysms: a natural history study. Neuroradiol 1999;441:338-41

17. Parkinson RJ, Eddleman CS, Batjer $\mathrm{HH}$, et al. Giant intracranial aneurysms: endovascular challenges. Neurosurgery 2008;62:1336-45

18. Gruber A, Killer M, Bavinski G, et al. Clinical angiographic results of endosaccular coiling treatment of giant and very large intracranial aneurysms: a 7-year, single-center experience. Neurosurgery 1999;45:793-803; discussion 803-04

19. Battaglia R, Pasqualin A, Da Pian R. Italian Cooperative Study on Giant Intracranial Aneurysms: Part 1-study design and clinical data. Acta Neurochir Suppl (Wien) 1988;42:49-52

20. Ding YH, Dai D, Danielson MA, et al. Control of aneurysm volume by adjusting the position of ligation during creation of elastase aneurysms: a prospective study. AJNR Am J Neuroradiol 2007;28:857-59

21. Ding Y, Dai D, Kadirvel R, et al. Creation of large elastase-induced aneurysms: presurgical arterial remodeling using arteriovenous fistulas. AJNR Am J Neuroradiol 2010;31:1935-37

22. Varsos V, Heros RC, DeBrun G, et al. Construction of experimental "giant" aneurysms. Surg Neurol 1984;22:17-20

23. Debrun GM, Varsos V, Liszczak TM, et al. Obliteration of experimental aneurysms in dogs with isobutyl-cyanoacrylate. J Neurosurg 1984;61:37-43

24. Kerber CW, Cromwell LD, Zanetti PH. Experimental carotid aneurysms: Part 2. Endovascular treatment with cyanoacrylate. Neurosurgery 1985;16:13-17

25. Yapor W, Jafar J, Crowell RM. One-stage construction of giant experimental aneurysms in dogs. Surg Neurol 1991;36:426-30

26. Anson JA. Epidemiology and natural history. In: Awad IA, Barrow AL, eds. Giant Intracranial Aneurysms. Park Ridge, IL: American Association of Neurological Surgeons; 1995:23-33

27. Graves VB, Ahuja A, Strother CM, et al. Canine model of terminal arterial aneurysm. AJNR Am J Neuroradiol 1993;14:801-03

28. Strother CM, Graves VB, Rappe A. Aneurysm hemodynamics: an experimental study. AJNR Am J Neuroradiol 1992;13:1089-95

29. Yasuda R, Strother CM, Taki W, et al. Aneurysm volume-to-ostium area ratio (VOR): a parameter useful in discriminating the rupture status of intracranial aneurysms. Neurosurgery $2011 ; 68: 310-18$

30. Black SP, German WJ. Observations on the relationship between the volume and the size of the orifice of experimental aneurysms. J Neurosurg 1960; 17:894-990

31. Hassan T, Timofeev EV, Saito T, et al. A proposed parent vessel geometrybased categorization of saccular intracranial aneurysms: computational flow dynamics analysis of the risk factors for lesion rupture. J Neurosurg 2005; 103:662-80

32. AbuRahma AF. Processes of care for carotid endarterectomy: surgical and anesthesia considerations. J Vasc Surg 2009;50:921-33 